Physician extenders

\section{Physician extenders in the emergency department}

\section{J R Hedges}

Data on cost-effectiveness and outcomes are required before physician extenders can become an integral part of the ED team in the UK health service

$\mathrm{S}$ mith and Tevis have provided a helpful comparative overview of the background, training, and practice environment of physician's assistants (PAs) working in US and UK emergency departments (EDs). ${ }^{1}$ However, they leave unanswered a number of questions about the role and relative value of PAs. This commentary highlights many of the issues that remain to be addressed and their wider implications.

As an emergency physician who has practised in EDs for nearly 30 years, I have witnessed the evolution of emergency medicine as a specialty in the USA and observed the more recent introduction of physician extenders (nurse practitioners (NPs) and PAs) in ED practice. $^{2-4}$ The notion of physician extenders has important implications for meeting workforce needs, specialisation within ED practice, and defining the specialty. The comments that follow are based on more than seven years of work with physician extenders in the Oregon Health and Science University (OHSU) ED and five years as a lecturer for the OHSU PA training programme.

\section{WHAT ARE "PHYSICIAN EXTENDERS"?}

The underlying concept of a physician extender is that of a non-medical healthcare provider who sees patients on behalf of or in conjunction with a lead physician or physician(s). This provider generally has a narrower knowledge base and skill set than the lead physician. In the USA, the commonest types of physician extender are PAs and NPs. The PA generally completes a 2+ year programme, including up to 14 months of clinical rotations, after receiving a college undergraduate degree. The NP generally completes a two year master's level course after a four year registered nurse degree programme. Clinical exposure is generally interspersed with didactic courses during the second year of the NP programme. The PA trainee's classes more closely resemble medical school classes and often are taught by the same physician faculty members who teach medical students. The emphasis of these didactic courses is upon applied clinical knowledge with a modicum of basic science. The NP trainee courses are generally taught by clinically experienced NPs who often put emphasis on nursing models of care delivery and also provide limited procedural training.

In both models, the trainee emerges with a knowledge base that has significantly less breath and depth than that of a newly qualified doctor. However, they will have key skills and knowledge in a narrow field. The depth of this skill set is dependent upon the trainee's clinical training opportunities and prior clinical exposure (which could be extensive if the trainee was a nurse or a paramedic).

The key message is that neither the hospital administrator nor the emergency medicine consultant should equate the physician extender with a fully trained specialist in a particular field of medicine, especially in the area of emergency medicine.

\section{WHAT IS THE ROLE OF THE PHYSICIAN EXTENDER IN THE ED?}

PAs and NPs generally serve in one or more roles:

- minor care provider

- extended care provider for clinically stable patients

- direct assistant to ED physician.

\section{Minor care provider}

Physician extenders (both PAs and NPs) most commonly deliver care by following protocols in settings where, through triage or an innate component of the overall care protocol, the high risk or more complex patients are directed to the consultant and/or registrar in the ED. Although this approach makes sense as a rational use of limited human resources, there are economic and quality related issues that we have only begun to address. We have much to learn about the relative efficiency of physician extenders in the ED with regard to patients requiring minor care (that is, the number of patients seen per hour per unit of salary for PAs $v$ house officers, general practitioners, registrars, or consultants).

Byrne and colleagues noted that NPs tended to process patients with minor injuries less rapidly, although the patients received more thorough instructions. ${ }^{5}$ Interestingly, when Sakr and colleagues compared their experience with an NP-led minor injury unit with similar care provided in their ED, the cost per case and frequency of postvisit follow up care were higher for patients seen in the NP-led minor injury unit. ${ }^{6}$ However, even if throughput (and resultant cost) efficiency is lower than when care is provided by doctors, physician extenders can provide vital extra staff during peak periods, evenings, weekends, and holidays, when minor care needs may be disproportionately high.

Similarly, we have limited information about the relative quality measures of physician extenders. Given that minor care patients should be carefully screened prior to evaluation by a physician extender, the inherent risk of an adverse outcome should be small. Sakr and colleagues noted that NPs and junior doctors had similar accuracy of examination, adequacy of treatment, planned follow up care, and requests for radiographs. ${ }^{7}$ The patients seen by NPs had fewer episodes of unplanned follow up for care advice. Cooper and colleagues also noted that patients with minor injuries had similar outcomes and greater patient satisfaction (associated with delivery of more injury related information) when seen by NPs than senior house officers. ${ }^{8}$

Thus, although the application of physician extenders for ED evaluation and management of well screened patients has been shown to be feasible, the relative efficiency of this practice and optimal minor illness or injury conditions remain to be determined.

\section{Extended care provider}

Although observation services are only beginning to evolve in the UK, more extended evaluation and management is common practice in the USA. Specialised ED wards deliver care that would traditionally require an inpatient admission. The use of physician extenders at OHSU Hospital began with the development of guidelines for management for common ED presentations requiring extended evaluation and management, and the concurrent 
development of a role for NPs to provide longitudinal care following established protocols. Conditions evaluated in the OHSU Hospital ED observation unit include undifferentiated chest pain, abdominal pain, dehydration, exacerbation of asthma/chronic obstructive pulmonary disease, deep venous thrombosis, mild diabetic ketoacidosis, closed head injury, and undifferentiated (haemodynamically stable) blunt torso trauma.

When providing longitudinal care in ED observation units, PAs and NPs operate within a narrow scope of practice that is highly focused on the longitudinal assessment of relatively stable patients while following carefully developed guidelines. In preparation for these roles, OHSU emergency physicians worked one to one with NPs to develop $\mathrm{NPs}^{\prime}$ assessment and interventional skills. ${ }^{9}$ Based upon national benchmark comparators for case-mix, the length of stay and admission rate data are quite good for this model. Ganapathy and Zwemer describe a similar model in which physician extenders provided continuity care in the ED pending transfer of relatively stable patients to a ward bed in the setting of delayed transfers to the ward. ${ }^{10}$ Further assessment will be needed to determine whether such care models are relevant for EDs in the UK.

\section{Assisting with the delivery of acute care}

The OHSU ED has initiated the codelivery of emergency care of more complicated and/or acutely ill/injured patients by physician extenders and faculty level (that is, consultant level) providers. Anecdotally, this experiment is a mixed success. It is clear that solo delivery of care to this patient population by physician extenders would be fraught with risk. Yet, in a setting where there are few house officers (for example, on a conference day), the physician extender can do much to support the efforts of the faculty physician. Indeed, Law and Sloan's survey suggested that a number of tasks performed by consultants could be delegated to a physician extender who would assist with (but not be responsible for) patient management. ${ }^{11}$ In the future, an analysis of added efficiency as a function of cost will need to be done. Further, the acceptance by PAs or NPs of a directly supportive role with limited independent decision making as a full time career is unknown.

\section{WHAT ARE THE WIDER IMPLICATIONS?}

Emergency medicine has been carved out of the acute care aspects of multiple clinical disciplines, often concentrating on those conditions that present an acutely evolving threat to a patient's life or limb. Despite this focus on acutely evolving illness or injury, EDs serve as portals for patients to access general medical care. Patients, and indeed other health professionals, cannot always determine whether their symptoms represent a serious health problem. Therefore, staff in EDs spend much of their time evaluating and managing less serious conditions. As Smith and Tevis suggest, physician extenders can provide much of the assessment of minor injury and minor illness. ${ }^{1}$ Although data evaluating relative efficiencies and quality of care are limited, years of experience suggest that physician extenders have an important role to play.

Were physician extenders to assume responsibility for all the less complex patient presentations, how would the consultant's practice be affected? Although the registrar may welcome this opportunity to forego the mundane in favor of the more challenging cases, the consultant may be less enamoured with a relentless body of complex cases from beginning to end of a clinical ED shift. For those who are 5+ years past their training, having an occasional straightforward case that can be assessed and resolved in 10-15 minutes can do much to "recharge one's batteries" during a clinical shift. Emergency physicians must think carefully about the practice environment they may create for themselves with the introduction and growth of physician extenders.

If the UK is to move towards greater use of physician extenders in the ED, what are the implications for the creation of PA training programmes in the UK? Are there special licensing concerns that must be addressed and practice restrictions or hospital credentialing limitations to be established? Establishment of the working relationship of the PAs with house staff, off-service consultants, and nurses will be critical.

It is difficult for me to ascertain whether the experiment reported by Smith and Tevis (see reference 1 ) is a true success. They do not detail the type of care provided by the PAs, the outcomes of that care, the cost of delivering care by PAs compared with other providers, or the acceptance of these physician extenders by patients or other hospital personnel. Yet, based upon the empirical application of physician extenders to emergency practice in American EDs, I anticipate that greater application of PAs will evolve in EDs in the UK, provided that the economics are favorable and the outcomes acceptable.

As this phenomenon evolves, I strongly encourage my colleagues in the UK to define carefully the PA's scope of practice and to make special effort to:

- provide close review of the practice on-site

- educate nurses, house staff, offservice consultants, and the public about the PA's scope of practice

- report efficiency and safety data associated with such a conjoint practice.

Emerg Med J 2005;22:314-315.

doi: 10.1136/emj.2004.022996

Correspondence to: Dr J R Hedges, Department of Emergency Medicine, CDW-EM, Oregon Health and Sciences University, 3181 SW Sam Jackson Park Rd, Portland, OR 97201-3098; USA; hedges¡@ohsu.edu

Competing interests: I have no conflict of interest or competing interest with this work other than the recent publication of a research project that is cited in the reference list for this article.

\section{REFERENCES}

1 Smith JS, Tevis B. Commentary from the fron lines: American physician assistants working in a United Kingdom emergency department. Emerg Med J 2005:22:322-4.

2 Sturmann KM, Ehrenberg K, Salzberg MR. Physician assistants in emergency medicine. Ann Emerg Med 1990;19:304-8.

3 Read SM, Jones NM, Williams BT. Nurse practitioners in accident and emergency departments: what do they do? BMJ 1992;305: 1466-70.

4 Hooker RS, McCaig L. Emergency department uses of physician assistants and nurse practitioners: a national survey. Am J Emerg Med 1996; 14:245-9.

5 Byrne G, Richardson M, Brunsdon J, et al. An evaluation of the care of patients with minor injuries in emergency settings. Accid Emerg Nurs 2000;8:101-9.

6 Sakr M, Kendall R, Angus J, et al. Emergency nurse practitioners: a three part study in clinical and cost effectiveness. Emerg Med J 2003;20:158-63.

7 Sakr M, Angus J, Perrin J, et al. Care of minor injuries by emergency nurse practitioners or junior doctors: a randomised controlled trial. Lancet 1999;354:1321-6.

8 Cooper MA, Lindsay GM, Kinn S, et al. Evaluating emergency nurse practitioner services: a randomized controlled trial. J Adv Nurs 2002;40:721-30.

9 Rondeau D, Norton RL, Vaughn K, et al. Model for inclusion of nurse practitioners in an academic department of emergency medicine [abstract]. Ann Emerg Med 2004:44:S131.

10 Ganapathy S, Zwemer FL Jr. Coping with a crowded ED: an expanded role for midlevel providers. Am J Emerg Med 2003;21:125-8.

11 Law H, Sloan J. Doctor's assistants - do we need them? J Accid Emerg Med 1999;16:114-16. 